\title{
L'HÉRÉdITÉ ET LA SÉCRÉTION DU LAIT
}

\author{
par \\ A. HOUDINIÈRE \\ Docteur-Vétérinaire sanitaire de la Seine.
}

Il y a peu d'années encore, la zootechnie nous apprenait que l'amélioration de la faculté laitière obtenue par la gymnastique fonctionnelle pouvait devenir à la longue un progrès définitif, désormais inscrit dans le patrimoine héréditaire des individus.

En d'autres termes la science de l'élevage admettait l'hérédité des caractères acquis.

Cette conception a été bouleversée par la génétique moderne dont les audacieuses théories ont reçu des confirmations expérimentales solides et nombreuses.

C'est à la lumière de ces acquisitions qu'il faut reconsidérer la question tout en cherchant à en dégager les conséquences pratiques dont peut bénéficier l'éleveur.

\section{$* * *$}

L'évolution des êtres vivants n'est pas une hypothèse, mais un fait solidement établi par un nombre infini de constatations positives que l'on doit à l'anatomie, à l'embryologie, à la paléontologie, à la physiologie, à la géonémie.

Elle relève de causes naturelles qui sont au moins au nombre de trois :

La variation de l'espèce ;

La concurrence pour vivre ;

La sélection, résultante des facteurs précédents.

En matière d'élevage, l'homme intervient pour faire disparaître la seconde de ces causes ou tout au moins l'atténuer et pour manier la troisième à son gré.

Les petites ou grandes différences que présentent entre eux les individus d'une même espèce sont dénommées variations. On distinguait autrefois les variations discontinues parce que rares et très éloignées du type normal (exemple: absence de mamelles, mamelles surnuméraires), et les variations continues qui présentent toutes sortes d'intermédiaires entre les variants extrêmes.

Mais ces distinctions ne donnaient aucune indication sur le matériel que la variation fournit à l'évolution.

Toutes les variations, en effet, ne sont pas héréditaires. La transmission de la vie est l'apanage d'un petit groupe de cellules dites sexuelles dont l'ensemble constitue le germen et que Weissmann a opposé radicalement à toutes les autres cellules du 
corps, beaucoup plus importantes par leur masse, et qui constituent le soma.

Il a été démontré expérimentalement qu'il y a une indépendance relative entre ces deux parties. C'est pourquoi on distingue maintenant deux sortes de variations :

1. La mutation qui, est une variation de la constitution physicochimique de la cellule sexuelle. Elle se traduit chez le descendant par une modification du soma et continue à se transmettre par son germen.

$2^{\circ}$ La somation, qui est la modification affectée par le soma seul d'un individu sous l'influence d'un changement dans les circonstances de milieu. On considère que cette variation n'a aucune raison de se transmettre aux descendants puisque le patrimoine héréditaire inscrit dans le germen reste inchangé.

Il existe cependant un lien entre le soma et le germen. Ce dernier est, en effet, imprégné par les sucs élaborés par le soma. Ce n'est donc que par l'intermédiaire de ces sucs que le germen pourrait être modifié.

Le grave problème est de savoir si une somation, caractère nouveau acquis par le soma seul, ne peut pas, lorsqu'il y a répétition de l'acquisition pendant de nombreuses générations (accroissement de la production du lait par la gymnastique fonctionnelle), se muer en mutation par une modification adéquate du patrimoine héréditaire.

\section{La mutation}

Avant d'examiner grosso modo ce que la génétique nous apprend sur la mutation, il faut étudier quelles sont les parties fondamentales de la cellule sexuelle et les qualités qu'on leur attribue.

Le noyau des cellules du corps pour une espèce donnée des êtres multicellulaires contient un nombre fixe de chromosomes ( $2 \mathrm{~N}$ ), éléments en grains ou rubans que l'on met en évidence par certains colorants. Ces corps sont groupés par paires contenant chacune un chromosome d'origine paternelle et un chromosome d'origine maternelle.

Lors de la multiplication de telles cellules, chaque chromosome se divise en deux parties égales de telle sorte que l'on retrouve toujours le même nombre de chromosomes dans les noyaux des deux nouvelles cellules formées $(2 \mathrm{~N})$.

Il n'en est plus de même lors de la formation des cellules sexuelles : le spermatozoïde et l'ovule.

Ici le noyau ne contient que la moitié des chromosomes primitifs (N) de sorte qu'au moment de la fécondation, l'œuf formé 
par l'union des gamètes mâle et femelle renferme le nombre normal de chromosomes ( $2 \mathrm{~N}$ ) particulier aux cellules de l'espèce.

Il faut cependant préciser que le phénomène a lieu suivant un processus assez compliqué (réduction numérique, disjonction qualitative) qui aboutit au fait qu'un seul chromosome de la même paire peut figurer dans le noyau de la cellule sexuelle cependant que cet élément peut être, suivant le hasard, d'origine maternelle ou paternelle.

L'équivalence manifeste qui existe entre les noyaux des gamètes a fait naître cette idée que les facteurs génétiques qui conditionnent l'apparition des caractères de l'individu siègent sur les chromosomes. Par la suite l'expérimentation est venue étayer cette hypothèse.

Dans la théorie chromosomique on identifie le chromosome visible avec une chaîne de facteurs génétiques qui sont enchaînés les uns aux autres (linkage) et ne se séparent qu'exceptionellement (crossing over).

L'ensemble des catégories de facteurs génétiques (désignés par des lettres conventionnelles) constitue pour un individu donné son génôme ou formule héréditaire.

La constitution interne et externe d'un être, telle qu'elle est conditionnée par son génôme, est appelée le génotype.

Le génotype influence directement ce que l'on appelle le phénotype, c'est-à-dire la somme des caractères extérieurement visibles d'un individu (1).

Mais cette influence n'est pas simple :

$1^{\circ}$ Tous les caractères en puissance dans les facteurs du génotype n'apparaissent pas sur le phénotype, Cela résulte de l'existence de facteurs dominants, dominés, léthals, etc., tels que les met en évidence l'application expérimentale des lois de Mendel;

$2^{\circ}$ S'il existe des caractères dits corrélatifs, conditionnés par un seul facteur (exemple le chat à yeux bleus est toujours sourd), il est aussi des caractères dont l'apparition dépend de facteurs multiples dont les actions s'additionnent ou se soustraient.

Il faut insistẹr sur ce dernier point parce qu'il intéresse la faculté laitière.

Certains caractères ne se transmettent pas en effet, dans toute leur intégrité. Ils présentent un certain degré d'indétermination ou d'oscillation. Considérons, par exemple, la longueur des oreilles du lapin : si l'on croise les deux types extrêmes, un lapin à oreilles longues avec un lapin à oreilles courtes, il n'y a pas dominance

(1) Nous verrons que le phénotype subit encore d'autres influences. 
d'un caractère sur l'autre; tous les hybrides obtenus ont des oreilles de longueur moyenne, oscillant autour de la moyenne arithmétique des longueurs parentales. Dans la seconde génération les longueurs intermédiaires seront encore plus nombreuses.

Mais si l'on sélectionne les lapins, en marchant vers les formes terminales, on obtient une majorité de lapins à oreilles longues ou à oreilles courtes suivant la dimension choisie.

Les caractères oscillants liés à des facteurs multiples, modifiables par sélection, sont fort nombreux.

L'aptitude laitière en est un exemple. La sélection pratiquée améliore le niveau moyen de production mais ne crée rien de nouveau. C'est une méthode d'isolement permettant d'obtenir à l'état de plus en plus pur la combinaison génotypique convenable. L'amélioration réalisée sera permanente, mais on ne pourra pas la dépasser. C'est ce qu'il convient d'appeler une population améliorée.

Que l'on cesse un choix sévère dans les reproducteurs, les oscillants supérieurs se mélangent aux inférieurs et l'on revient à un état intermédiaire à peu près stable.

Un bel exemple d'orthogénèse déterminée par sélection est celui de l'augmentation de la quantité de lait fournie par les brebis du Larzac. En 1875, ces femelles donnaient annuellement une quantité de lait suffisante pour fabriquer 6 kilogrammes de fromage de Roquefort; actuellement, ces brebis à mamelles monstrueuses en produisent assez pour fabriquer 14 à 15 kilogrammes de cet aliment (1).

On soutient maintenant que la faculté laitière est fonction au moins de trois facteurs génétiques qui correspondraient :

A l'innervation des trayons ;

A la puissance de l'appareil digestif ;

A la valeur fonctionnelle des glandes endoerines (GINIEIS) (2).

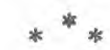

Ainsi le génotype, indépendant des agents extérieurs est héréditaire et inaltérable. Est-ce à dire qu'un autre génotype permanent ne puisse en dériver? Non. Il suffit pour cela que l'un de ses facteurs ou l'une de ses catégories subisse une perturbation profonde.

Ceci caractérise la mutation qui apparaît comme un changement dans une très petite région définie du chromosome.

(1) A cette sélection incessante s'ajoutent les effets non héréditaires de l'entraîne ment et du régime approprié.

(2) J. Grinreis. La génétique. Ses applications aux phénomènes de l'hérédité Cahiers de Médecine Vétérinaire, 1942, $15^{\mathrm{e}}$ année, no $7,8,9$. 
C'est d'ailleurs la mutation qui permet de déterminer l'existence des facteurs génétiques. Les diverses catégories de facteurs ont présenté au cours des âges des mutations en nombre variable ; tel facteur n'en a qu'une, tandis que tel autre en a présenté trois...

Le nombre de génotypes possibles dans une espèce donnée peut être calculé d'après le nombre de catégories de facteurs actuellement connues et le nombre de mutations que chacun d'eux a présentées.

Dans ce calcul on arrive rapidement à des chiffres astronomiques. L'espèce souris par exemple, chez laquelle on ne connaît. que peu de mutations pourrait fournir 5.242 .800 génotypes différents, parmi lesquels on a obtenu expérimentalement tous ceux que l'on a voulu réaliser.

La mutation fournit donc un matériel formidable à l'évolution d'une espèce moyennement variable.

Il en résulte qu'en matière d'aptitude laitière et beurrière il faut toujours attacher un intérêt marqué aux animaux d'élite dont la production extraordinaire peut correspondre à un génotype nouveau. Cette attention doit se porter non seulement sur les animaux sévèrement sélectionnés, mais aussi sur le bétail courant qui tout autant que le premier. peut être l'objet de mutation.

Le génotype nouveau étant soupçonné, son diagnostic sera confirmé par l'application des lois bien connues de Mendel (découverte de l'homozygote par l'isolement des récessifs et le croisement de retour, accouplement des homozygotes...). Au surplus, à défaut de connaissances analytiques, n'importe quel praticien peut accumuler les facteurs productifs dans une même famille par la sélection et la consanguinité.

Tout ceci montre de plus tout l'intérêt qu'il faut attacher à la généralisaton du contrôle laitier dont les résultats interprétés par famille et dans le temps autorisent l'application des données précédentes, c'est-à-dire l'obtention d'une amélioration raciale de la population considérée.

\section{La somation}

Lorsqu'un changement dans les circonstances de milieu affectera le soma seul d'un individu sans toucher au germen, l'animal présentera une modification plus ou moins importante, qui n'aura aucune raison de se transmettre à ses descendants, puisque par définition le patrimoine héréditaire reste inchangé. Ce sera une somation.

C'est un phénomène très général que possède les tissus des métazoaires de réagir d'une façon adaptative, d'un bout de leur vie 
à l'autre, à un grand nombre d'excitations qu'on peut appeler normales.

Cette propriété régulatrice intervient dans le jeune âge d'une façon capitale pour construire et adapter le corps aux conditions du milieu ; plus tard elle lui permet de s'adapter au mieux lorsque les conditions de l'existence offrent de légers changements ou de revenir à un état d'équilibre physiologique lorsque celui-ci a été momentanément troublé.

Le tableau ci-dessous résume les réponses utiles à un certain nombre d'événements (1) :

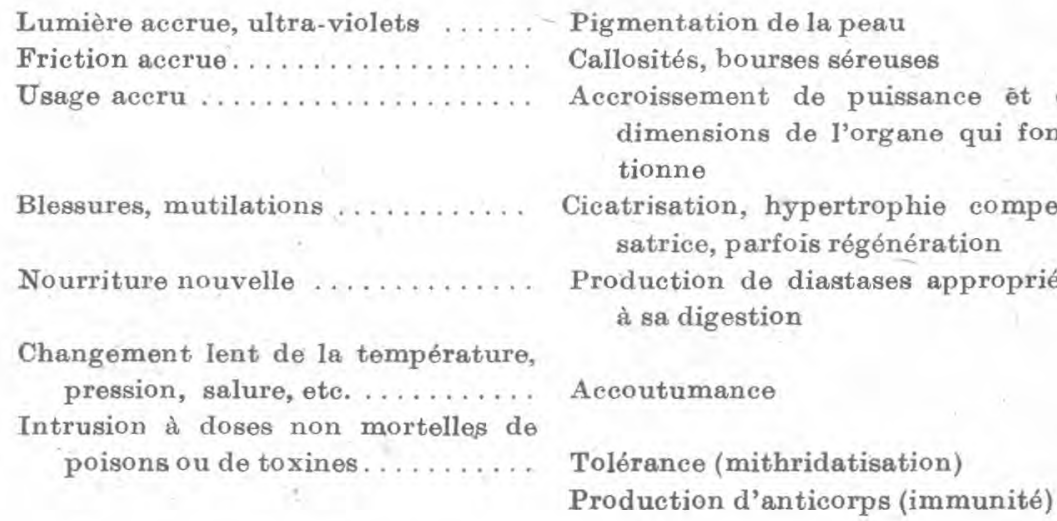

On explique cette force coordinatrice de l'organisme par les communications nerveuses et par l'action des hormones.

La vie serait inconcevable si, dès le début, les êtres n'avaient pas possédé ces réactions utiles.

Cependant, la force qui les dirige ne semble pas parfaite. Ses erreurs et surtout ses caprices sont innombrables Il faut donc accepter les réactions d'adaptation fonctionnelle comme données ; elles sont souvent utiles, parfois indifférentes, quelquefois nuisibles ; leur étendue est très variable, ce qui joue un rôle prépondérant dans l'extension des métazoaires.

On a appelé plastotype la modification produite sur le soma par le milieu, de sorte que l'on peut écrire l'égalité suivante : plastotype + génotype = phénotype.

L'amélioration de la production du lait par les conditions ambiantes favorables est un exemple de somations voulues par l'homme. C'est en agissant sur le milieu, sur la gymnastique fonctionnelle et l'alimentation que l'on obtient des résultats remarquables.

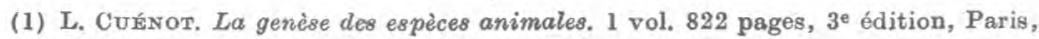
1932. Félix Alean, éditeur, 108, boulevard St-Germain, Paris, VI. 
Mais la question qui se pose, comme pour toutes les autres adaptations somatiques, est de savoir si ces résultats sont définitivement acquis; e'est de savoir si l'accomodat obtenu répété pendant des années s'inscrit dans le patrimoine héréditaire de l'espèce (sous la forme d'un facteur du génotype).

C'était là la conception admise à la fin du xıx ${ }^{\text {e }}$ siècle. Au premier rang des facteurs de l'évolution des espèces, on plaçait l'action $\mathrm{du}$ milieu, agent principal de modification des organismes.

A la suite des Buffon, des Lamarck et des Geoffroy, on admettait qu'une adaptation temporaire, entretenue pendant un temps suffisant, finissait par s'insérer dans le patrimoine héréditaire et devenait un caractère fixe et stable, désormais indépendant de la cause extérieure qui, chez les ancêtres, en avait amené la naissance.

Dans son Traité de Zootechnie Générale, en 1928, DеснамвRе (1) écrivait encore : "C'est par ce mécanisme (gymnastique fonctionnelle de la mamelle) que se sont formées les races grandes laitières de vaches, de brebis et de chèvres, chez lesquelles la glande mammaire présente un volume beaucoup plus considérable que chez les races incultes ou mauvaises laitières. "

Mais c'est ce passage du temporaire au définitif, ce transfert au patrimoine de l'espèce du caractère acquis par l'individu au cours de sa vie qu'il est difficile d'accepter dans l'état actuel de nos connaissances sur les cellules germinales et leurs rapports avec le soma. C'est pourquoi Wmismann et son école mutationiste le nient formellement.

Cependant, seules des preuves expérimentales peuvent apporter des certitudes en la matière. Dans son livre magistral, Cú́noT s'est efforcé d'examiner le problème sous ce jour.

Il répond en affirmant que les expériences, dans les limites où l'homme peut expérimenter, sont nettement contraires à l'hypothèse de l'inscription patrimoniale plus ou moins immédiate des caractères acquis par le soma.

Les explications des Lamarckistes sont toujours susceptibles d'autres interprétations parfois plus vraisemblables.

Certes, tout se passe comme s'il y avait une relation causale entre le climat et les caractères héréditaires des races géographiques, entre le froid et la fourrure blanche des animaux polaires, entre l'obscurité et la cécité des animaux cavernicoles, etc., mais le processus Lamarckien est inconcevable et on doit croire à l'inter.

(1) P. Dechambre. Traité de Zootechnie, t. I (Zootechnie générale), 4e édition, Paris, 1928. Librairie agricole de la Maison Rustique, 26, rue Jacob, Paris, VI. 
vention d'autres facteurs comme la préadaptation, la sélection naturelle, sans compter les facteurs inconnus.

Pour cet auteur, les somations peuvent avoir un très grand intérêt en permettant à certaines espèces de vivre dans des milieux nouveaux, mais ce ne sont que des sortes d'épiphénomènes qui n'ont eu aucune influence sur l'évolution des espèces.

On ne peut ici, entrer dans le détail des arguments apportés. Mais il faut ajouter que la majorité des généticiens modernes et des grands zootechniciens se sont ralliés à cette conception puisque l'expérimentation n'a pu trouver un seul eas authentique de transmissibilité d'un caractère acquis.

En matière d'élevage, on peut donc dire que lorsqu'une modification soi-disant acquise, causée par les milieux ou l'entraînement (aptitude laitière) se montre héréditaire, c'est qu'une erreur d'interprétation travestit la nature de l'évolution.

Il ne s'agit pas d'un earactère acquis, mais d'une sélection, d'une orthogénèse de caractères oscillants supérieurs aux caractères moyens, d'un earactère récessif réapparu, d'un facteur génétique occulte enfin réalisé, d'une ou de mutations passées inaperȩues.

$$
* * *
$$

L'action des agents extérieurs sur les phénotypes complique done le problème de la transmission des caractères. Aussi est-il difficile de distinguer les rôles respectifs des facteurs génétiques et des facteurs extérieurs.

Pour écarter l'influence du génotype on peut examiner les somations sur des sujets étroitement apparentés, c'est-à-dire possédant la même formule héréditaire. A ce sujet les jumeaux univitellins sont d'un grand secours.

Les animaux seront alors mis dans des conditions de vie différentes de celles où ils se trouvent sans dépasser leurs limites de résistance.

C'est ce qui se produit par exemple pour les animaux domestiques qui ont repris la vie libre (ou marrons), comme les vaches de l'Amérique du Sud que l'on cesse de traire et dont la mamelle régresse, perdant ainsi les caractères acquis en domesticité.

Inversement pour éliminer l'influence du plastotype, il faut faire porter les contrôles pendant la vie entière des individus et placer les animaux à comparer dans les mêmes conditions de milieu.

En résumé, on peut déduire des connaissances que nous apporte 
la génétique contemporaine que le perfectionnement de l'aptitude laitière de nos animaux est liée à trois ordres, d'améliorations :

10 L'obtention de combinaisons génotypiques à rendement plus élevé que le rendement moyen de la population considérée ;

$2^{\circ}$ La recherche des mutations en vue de perpétuer les génotypes nouveaux dont le rendement est supérieur aux précédents;

$3^{\circ}$ Le maintien permanent de l'influence des agents extérieurs favorables aux améliorations non héréditaires du soma.

A l'encontre de ce que l'on croyait autrefois, les perfectionnements obtenus par l'action des milieux ou de la gymnastique fonctionnelle ne sont pas acquis pour la race ou l'espèce et disparaissent dès que cessent les conditions favorables qui leur ont donné naissance.

Les méthodes de reproduction doivent tenir compte de ces notions pour tendre non seulement vers des rendements plus élevés, mais aussi vers une stabilité plus grande des améliorations obtenues grâce au perfectionnement du potentiel héréditaire des animaux.

\title{
REVUE
}

\section{L'INDUSTRIE LAITIERE A L'ÉTRANGER}

\author{
par \\ G. GÉNIN \\ Ingénieur E. P. C.

\section{ÉTATS-UNIS}

\section{Fabrication de la fibre de caséine}

La National Dairy Products Corporation a entrepris, au cours, du deuxième semestre 1941 la fabrication de la laine de lait. Ce produit, qui porte le nom d'Aralac est dû aux travaux de l'Atlantic Research Association Inc. Une fabrique d'essai a tout d'abord été mise en service à Bristol, dans te Rhode Island, puis l'Aralac Inc., a construit à Taftville, dans le Connecticut, une fabrique qui, travaillant 24 heures par jour, est susceptible de produire 2.300 tonnes d'Aralac par an. Pour assurer cette production, l'usine utilisera 72.000 tonnes de lait écrémé.

La fibre Aralac est plus chère que le coton ou la soie artificielle, mais son prix est inférieur à celui de la laine. 